\title{
Matrix Elements of Representations of Non-Compact Groups in a Continuous Basis*
}

\author{
A. O. Barut and E. C. Phillips \\ Department of Physics and Department of Mathematics, \\ University of Colorado
}

Received December 1, 1967

\begin{abstract}
Explicit formulas are obtained by a simple algebraic method for the representations of the finite group transformations of $O(2,1)$ in a continuous basis when a non-compact generator is diagonalized. Compact and non-compact cases are treated in a unified form and the nature of analytic continuation is determined. The transformation function between the discrete and the continuous bases is also given. These explicit formulas have not been obtained in the literature before.
\end{abstract}

\section{Introduction}

The use of the unitary infinite-dimensional representations of noncompact groups to describe the properties of bound states of quantummechanical systems is by now well understood. However, not much has been done to deal with the scattering states. There is a need for explicite forms of unitary representations when a continuous spectrum is used to label the states (i.e. diagonalized). There arise here some peculiar and unfamiliar (at least to physicists) problems that must be solved.

There has been a number of recent discussions on the unified representation theory of compact and non-compact groups having the same complex extention [1-3]. A number of recent papers deal with the specific cases of the representations of $O(3,1)$ with respect to the noncompact group $O(2,1)$ and the analytic continuation problem between $O(2,1)$ and $O(3)[1-3,8-10]$. In none of the previous work there appears the explicite form of the representation in a continuous basis and the relation of the continuous basis to the discrete one. The purpose of this work is to fill this gap. The simple algebraic method that we give in this paper as an extension of the previous work [3] not only determines the representations in a continuous basis $|\lambda\rangle$, but also gives explicite formulae for the transformation function $\langle m \mid \lambda\rangle$ between the discrete basis $|m\rangle$ and the continuous basis $|\lambda\rangle$, and the matrix elements $\left\langle\lambda|U| \lambda^{\prime}\right\rangle$, where

* Supported in part by the Air Force Office of Scientific Research, Office of Aerospace Research, U.S. Air Force, under Grant No. AF-AFOSR- 30-67. 
$U$ is any group element. These are the quantitites of prime interest in physical applications. We confine ourselves to the simple prototype case, namely the group $O(2,1)$, but the results can easily ge generalized to higher groups.

In Section II we derive as a preliminary the unitary representations of $O(2,1)$ from those of $O(3)$ by analytic continuation. This is a correspondence between two unitary representations, and of course, is different than the usual correspondence of finite dimensional unitary representation of $O(3)$ and the finite dimensional non-unitary representation of $O(2,1)$. In Section III, the main part of the paper, we introduce the continuous basis by a suitable redefinition of the creation and annihilation operators and derive the above mentioned quantities $\langle m \mid \lambda\rangle$ and $\left\langle\lambda|U| \lambda^{\prime}\right\rangle$. The nature of analytic continuation from the discrete basis to the continuous basis is of course not unique and straightforward as one might think, and special attention is given to this problem.

\section{Collection of Formulas. Derivation of Unitary $O(2,1)$ - Representations by Analytic Continuation}

\section{(1) Lie Algebras}

The elements of the Lie algebras of $O(3)-O(2,1)$ system satisfy [3]

$$
\begin{aligned}
& {\left[L_{12},\right.}\left.L_{13}\right]=i L_{12} \\
& {\left[L_{23},\right.}\left.L_{13}\right]=i g_{33} L_{12} \\
& {\left[L_{23},\right.}\left.L_{12}\right]=i L_{13} \\
& g_{33}=-1 \text { for } O(3), g_{33}=+1 \text { for } O(2,1),
\end{aligned}
$$

or, in canonical form, with $L^{ \pm} \equiv \frac{1}{\sqrt{2}}\left(L_{13} \pm i L_{23}\right)$,

$$
\begin{aligned}
{\left[L_{12}, L^{ \pm}\right] } & = \pm L^{ \pm} \\
{\left[L^{+}, L^{-}\right] } & =-g_{33} L_{12} .
\end{aligned}
$$

Thus, starting with the $O(3)$ algebra, $L_{12} L_{13}, L_{23}$, the $O(2,1)$ algebra can be obtained by the new elements

$$
\bar{L}_{12}=L_{12}, \bar{L}_{13}=i L_{13}, \bar{L}_{23}=i L_{23} \text {. }
$$

\section{(2) Fundamental Representation of the Groups}

The spinor representations of the group $O(3)$ and $O(2,1)$ (not algebras!) are given by [3]

$$
W=\left(\begin{array}{ll}
\alpha & \beta \\
g_{33} \bar{\beta} & \bar{\alpha}
\end{array}\right), \operatorname{det} W=1 ;\left\{\begin{array}{lll}
g_{33}=-1 & \text { for } & O(3) \\
g_{33}=+1 & \text { for } & O(2,1)
\end{array},\right.
$$


or, we can introduce real Euler angles parameters such that

$$
\begin{gathered}
W=\left(\begin{array}{ll}
e^{i \mu / 2} & \\
& e^{-i \mu / 2}
\end{array}\right)\left(\begin{array}{rr}
\cos (\varepsilon \xi / 2) & \sin (\varepsilon \xi / 2) \\
-\sin (\varepsilon \xi / 2) & \cos (\varepsilon \xi / 2)
\end{array}\right)\left(\begin{array}{ll}
e^{i v / 2} & \\
& e^{-i \nu / 2}
\end{array}\right), \\
\alpha=e^{i(\mu+\nu) / 2} \cos (\varepsilon \xi / 2) ; \quad \beta=e^{i(\mu-v) / 2} \sin (\varepsilon \xi / 2), \quad \varepsilon=+\sqrt{-g_{33}} .
\end{gathered}
$$

This decomposition of $W$ corresponds to the decomposition of the group element

$$
W=e^{i \mu L_{12}} e^{i \xi L_{28}} e^{i \nu L_{12}},
$$

and to the representation of the algebra

$$
L_{12}=\frac{1}{2} \sigma_{3}, \quad L_{13}=\frac{\varepsilon}{2} \sigma_{1}, \quad L_{23}=\frac{\varepsilon}{2} \sigma_{2}, \quad \varepsilon=+\sqrt{-g_{33}} .
$$

\section{(3) The General Linear Representation}

From (2.5) or (2.6) the matrix elements of a general representations are of the form

$$
\mathscr{D}(\mu \xi \nu)_{m^{\prime} m}=e^{i m^{\prime} \mu} d(\xi)_{m^{\prime} m} e^{i m \nu} .
$$

Thus it is sufficient to find $d(\xi)_{m^{\prime} m}$ corresponding to the group element

$$
\begin{aligned}
U & =\left(\begin{array}{rr}
\cos (\varepsilon \xi / 2) & \sin (\varepsilon \xi / 2) \\
-\sin (\varepsilon \xi / 2) & \cos (\varepsilon \xi / 2)
\end{array}\right)=\left(\begin{array}{rr}
\alpha & \beta \\
g_{33} \bar{\beta} & \bar{\alpha}
\end{array}\right) \\
U^{-1} & =\left(\begin{array}{rr}
\bar{\alpha} & -\beta \\
-g_{33} \bar{\beta} & \alpha
\end{array}\right) .
\end{aligned}
$$

We determine the representations induced on the basis functions [3]

$$
|\Phi, m\rangle=A_{m} X^{\Phi+m} Y^{\Phi-m},
$$

where $\Phi$ and $m$ are for the moment arbitrary complex numbers, and $A_{m}$ suitable normalization constants. Then

$$
\mathscr{D}(W)|\Phi, m\rangle=A_{m}[\bar{\alpha} X-\beta Y]^{\Phi+m}\left[-g_{33} \bar{\beta} X+\alpha Y\right]^{\Phi-m} .
$$

For $0<|\beta|<|\alpha|$, i.e. $|\xi / 2|<\pi / 2$, we can expand the bracket by the binomial theorem:

$$
\begin{aligned}
& \mathscr{D}(W)|\Phi, m\rangle=A_{m} \sum_{N=0}^{\infty}\left(\begin{array}{c}
\Phi+m \\
N
\end{array}\right) \\
& \cdot(-\beta Y)^{N}(\bar{\alpha} X)^{\Phi+m-N} \sum_{N^{\prime}=0}^{\infty}\left(\begin{array}{c}
\Phi-m \\
N^{\prime}
\end{array}\right)\left(-g_{33} \bar{\beta} X\right)^{N^{\prime}}(\alpha Y)^{\Phi-m-N^{\prime}} .
\end{aligned}
$$

The series converges absolutely; thus we can rearrange terms:

$$
\begin{aligned}
& \mathscr{D}(W)|\Phi, m\rangle=A_{m} \sum_{N, N^{\prime}=0}^{\infty}\left(\begin{array}{c}
\Phi+m \\
N
\end{array}\right)\left(\begin{array}{c}
\Phi-m \\
N^{\prime}
\end{array}\right) \\
& \cdot(-\beta)^{N}\left(-g_{33} \bar{\beta}\right)^{N^{\prime}} \bar{\alpha}^{\Phi+m-N} \alpha^{\Phi-m-N^{\prime}} X^{\Phi+m-N+N^{\prime}} Y^{\Phi-m-N^{\prime}+N} .
\end{aligned}
$$


Now let $m^{\prime}=m-N+N^{\prime}$, then for $m>m^{\prime}$ :

$$
\begin{aligned}
\mathscr{D}(W)|\Phi, m\rangle= & \sum_{m^{\prime}} \frac{A_{m}}{A_{m^{\prime}}} \sum_{N^{\prime}=0}^{\infty}(-\beta)^{m-m^{\prime}+N^{\prime}}\left(-g_{\mathbf{3} 3}\right)^{N^{\prime}} \bar{\beta}^{N^{\prime}} \bar{\alpha}^{\Phi+m^{\prime}-N^{\prime}} \\
& \cdot \alpha^{\Phi-m-N^{\prime}}\left(\begin{array}{c}
\Phi+m \\
m-m^{\prime}+N^{\prime}
\end{array}\right)\left(\begin{array}{c}
\Phi-m \\
N^{\prime}
\end{array}\right)\left|\Phi, m^{\prime}\right\rangle \\
= & \sum_{m^{\prime}} \frac{A_{m}}{A_{m^{\prime}}} \bar{\alpha}^{\Phi+m^{\prime}} \alpha^{\Phi-m}(-\beta)^{m-m^{\prime}} \sum_{N^{\prime}}\left(\begin{array}{c}
\Phi+m \\
m-m^{\prime}+N^{\prime}
\end{array}\right) \\
& \cdot\left(\begin{array}{c}
\Phi-m \\
N^{\prime}
\end{array}\right)\left(g_{\mathbf{3} 3}\right)^{N^{\prime}}\left(\frac{\beta \bar{\beta}}{\alpha \bar{\alpha}}\right)^{N^{\prime}}\left|\Phi, m^{\prime}\right\rangle .
\end{aligned}
$$

Or, introducing the hypergeometric function, we get for the matrix elements

$$
\begin{aligned}
& \mathfrak{S}(W)_{m^{\prime} m}=\frac{A_{m}}{A_{m^{\prime}}} \frac{\bar{\alpha}^{\Phi+m^{\prime}} \alpha^{\Phi-m}(-\beta)^{m-m^{\prime}}}{\left(m-m^{\prime}\right) !} \frac{(\Phi+m) !}{\left(\Phi+m^{\prime}\right) !} \\
& \cdot F\left(-\Phi-m^{\prime},-\Phi+m, 1+m-m^{\prime} ; g_{33} \frac{|\beta|^{2}}{|\alpha|^{2}}\right) ; m>m^{\prime} ;|\beta|<|\alpha| .
\end{aligned}
$$

For $m^{\prime}>m,|\beta|<|\alpha|$, we sum on $N$ rather than $N^{\prime}$ in the original expression

$$
\begin{aligned}
\mathscr{D}(W)|\Phi, m\rangle= & \sum_{m^{\prime}} \frac{A_{m}}{A_{m^{\prime}}} \sum_{N}(-\beta)^{N}\left(-g_{33} \bar{\beta}\right)^{m^{\prime}-m+N}(\bar{\alpha})^{\Phi+m-N} \alpha^{\Phi-m^{\prime}-N} \\
& \cdot\left(\begin{array}{c}
\Phi+m \\
N
\end{array}\right)\left(\begin{array}{c}
\Phi-m \\
m^{\prime}-m+N
\end{array}\right)\left|\Phi, m^{\prime}\right\rangle \\
= & \sum_{m^{\prime}} \frac{A_{m}}{A_{m^{\prime}}}(\bar{\alpha})^{\Phi+m} \alpha^{\Phi-m^{\prime}}\left(-g_{33} \bar{\beta}\right)^{m^{\prime}-m} \sum_{N}\left(g_{33} \frac{|\beta|^{2}}{|\alpha|^{2}}\right)^{N} \\
& \cdot\left(\begin{array}{c}
\Phi+m \\
N
\end{array}\right)\left(\begin{array}{c}
\Phi-m \\
m^{\prime}-m+N
\end{array}\right)\left|\Phi, m^{\prime}\right\rangle .
\end{aligned}
$$

Thus, for $m^{\prime} \geqq m,|\beta|<|\alpha|$

$$
\begin{aligned}
\mathfrak{S}(W)_{m^{\prime} m}= & \frac{A_{m}}{A_{m^{\prime}}} \frac{(\bar{\alpha})^{\Phi+m}(\alpha)^{\Phi-m^{\prime}}\left(-g_{\mathbf{3} 3} \bar{\beta}\right)^{m^{\prime}-m}}{\left(m^{\prime}-m\right) !} \frac{(\Phi-m) !}{\left(\Phi-m^{\prime}\right) !} \\
& \cdot F\left(-\Phi+m^{\prime},-\Phi-m, 1+m^{\prime}-m, g_{33} \frac{|\beta|^{2}}{|\alpha|^{2}}\right)
\end{aligned}
$$

In all the above equations we have actually

$$
\begin{aligned}
& m \rightarrow E_{0}+m \\
& m^{\prime} \rightarrow E_{0}+m^{\prime},
\end{aligned}
$$

where $E_{0}$ is the fractional part of $m$, and is fixed.

Let

and use

$$
\frac{z}{z-1}=g_{33} \frac{|\beta|^{2}}{|\alpha|^{2}}, z=-g_{33}|\beta|^{2}
$$

$$
(1-z)^{a} F(a, b, c ; z)=F(a, c-b, c ; z /(z-1))
$$


then

$$
\begin{aligned}
\mathfrak{S}(W)_{m^{\prime} m}= & \frac{A_{m}}{A_{m^{\prime}}} \frac{(\Phi+m) !}{\left(\Phi+m^{\prime}\right) !} \frac{\alpha-m-m^{\prime}(-\beta)^{m-m^{\prime}}}{\left(m-m^{\prime}\right) !} \\
& \cdot F\left(-\Phi-m^{\prime}, 1+\Phi-m^{\prime}, m-m^{\prime}+1,-g_{33}|\beta|^{2}\right) ; m \geqq m^{\prime} \\
D(W)_{m^{\prime} m}= & \frac{A_{m}}{A_{m^{\prime}}} \frac{(\Phi-m) !}{\left(\Phi-m^{\prime}\right) !} \frac{\bar{\alpha}^{m+m^{\prime}}\left(-g_{33} \bar{\beta}\right)^{m^{\prime}-m}}{\left(m^{\prime}-m\right) !} \\
& \cdot F\left(-\Phi+m^{\prime}, \Phi+m^{\prime}+1 ; m^{\prime}-m+1 ;-g_{33}|\beta|^{2}\right) ; m^{\prime} \geqq m .
\end{aligned}
$$

\section{(4) Irreducible Representations}

Up to now $\Phi$ and $m$ were arbitrary complex numbers. Now we find the ranges of $\Phi$ and $m$ for irreducible representations.

To find irreducible subspaces we find a subset $M$ of the $m$ 's such that for $m \in M, D_{m^{\prime} m}=0$, if $m^{\prime} \notin M$, for every $\beta$. Because $A_{m} \neq 0$ in any irreducible subspace, the only way $D_{m^{\prime} m}$ can vanish is for

$$
\begin{aligned}
& \frac{(\Phi+m) !}{\left(\Phi+m^{\prime}\right) !}=0, m \geqq m^{\prime} \\
& \frac{(\Phi-m) !}{\left(\Phi-m^{\prime}\right) !}=0, m^{\prime} \geqq m .
\end{aligned}
$$

(A) If $(\Phi+m)$ and $(\Phi-m)$ are not integers, then $D_{m^{\prime} m} \neq 0$ for all $m$ and the representation is irreducible. We can require $-1 / 2$ $\leqq \operatorname{Re} E_{0} \leqq 1 / 2$ and the corresponding representations are denoted by $\mathscr{D}\left(Q, E_{0}\right), Q=\Phi(\Phi+1)$.

(B) If $(\Phi+m)$ is integer, $(\Phi-m$ not), then the subspace $m \geqq-\Phi$ is invariant, since for any $m^{\prime}<-\Phi \leqq m, \Phi+m^{\prime}$ is a negative integer. The representation is $\mathscr{D}^{+}(\Phi), E_{0}=-\Phi, m \geqq-\Phi$.

(C) If $\Phi-m=$ integer $(\Phi+m$ not), similarly $\Phi \geqq m$ is an invariant subspace: $\mathscr{D}-(\Phi), E_{0}=\Phi, m \leqq \Phi$.

(D) $\Phi+m, \Phi-m$ both integers :

(i) $\Phi<0$, we again get $\mathscr{D}^{+}$or $\mathscr{D}^{-}$.

(ii) $\Phi \geqq 0$ the irreducible subspace is finite dimensional, $-\Phi \leqq m \leqq \Phi$.

\section{(5) Unitary Representations}

The fundamental representation preserves the form

Hence

$$
|X|^{2}-g_{33}|Y|^{2} \text {. }
$$

$$
\begin{aligned}
{[(2 \Phi) !]^{-1} } & \left\{|X|^{2}-g_{33}|Y|^{2}\right\}^{2 \Phi} \\
& =[(2 \Phi) !]^{-1}\left\{|\bar{\alpha} X-\beta Y|^{2}-g_{33}\left|-g_{33} \bar{\beta} X+\alpha Y\right|^{2}\right\}^{2 \Phi}
\end{aligned}
$$

(the factor $[(2 \Phi) !]^{-1}$ is for convenience only). Expanding both sides of 
this equation we get

$$
\begin{aligned}
& \sum_{N=0}^{\infty} \frac{\left(-g_{33}\right)^{N}}{N !(2 \Phi-N) !}|X|^{2 N}|Y|^{2(2 \Phi-N)} \\
= & \sum_{N=0}^{\infty} \frac{\left(-g_{33}\right)^{N}}{N !(2 \Phi-N) !}|\bar{\alpha} X-\beta Y|^{2 N}\left|-g_{33} \bar{\beta} X+\alpha Y\right|^{2(2 \Phi-N)} .
\end{aligned}
$$

We wish to identify these expansions with the sum of squares of (2.9)

$$
\begin{aligned}
& \sum_{m}\left|A_{m}\right|^{2}|X|^{2(\Phi+m)}|Y|^{2(\Phi-m)} \\
= & \sum_{m}\left|A_{m}\right|^{2}|\bar{\alpha} X-\beta Y|^{2(\Phi+m)}\left|-g_{33} \bar{\beta} X+\alpha Y\right|^{2(\Phi-m)} .
\end{aligned}
$$

In the case of $\mathscr{D}^{+}(\Phi)$ representations this can be done, if we change the indices $N$ in (2.16) to $\Phi+m$ (actually to $\Phi+E_{0}+m$ ) and sum over $m$ from $-\Phi$ to $\infty$. Thus, we have

or,

$$
0 \leqq\left|A_{m}\right|^{2}=\frac{\left(-g_{33}\right)^{\Phi+m}}{(\Phi+m) !(\Phi-m) !}
$$

$$
0 \leqq \frac{\left|A_{m+1}\right|^{2}}{\left|A_{m}\right|^{2}}=\left(-g_{33}\right) \frac{\Phi-m}{\Phi+m+1},
$$

which agrees with Eq. (1.30) of Ref. 3. The ranges of $\Phi$ and $m$ are determined by Eq. (2.19) and agree with the known results $[8,10]$ which are now determined by an analytical continuation.

\section{Continuous Basis}

The representation of the Lie algebra corresponding to (2.9) is in terms of the boson creation and annihilation operators

$$
\begin{aligned}
& L_{12}=\frac{1}{2}\left(a_{1}^{*} a_{1}-a_{2}^{*} a_{2}\right) \\
& L_{13}=\frac{\varepsilon}{2}\left(a_{1}^{*} a_{2}+a_{2}^{*} a_{1}\right) \\
& L_{23}=-i \frac{\varepsilon}{2}\left(a_{1}^{*} a_{2}-a_{2}^{*} a_{1}\right),
\end{aligned}
$$

or,

$$
\begin{aligned}
& L^{+}=\frac{\varepsilon}{\sqrt{2}} a_{1}^{*} a_{2} \\
& L^{-}=\frac{\varepsilon}{\sqrt{2}} a_{2}^{*} a_{1} .
\end{aligned}
$$

This representation is suitable for the diagonalization of $L_{\mathbf{1 2}}$. Suppose we want to diagonalize $L_{23}$ which has a continuous spectrum for the noncompact case: $g_{33}=1$. For this purpose we define a new set of boson 
operators

$$
c_{1}^{*}=\frac{1}{\sqrt{2}}\left(a_{1}^{*}+i a_{2}^{*}\right), \quad c_{2}^{*}=\frac{1}{\sqrt{2}}\left(a_{2}^{*}+i a_{1}^{*}\right)
$$

or,

$$
a_{1}=\frac{1}{\sqrt{2}}\left(c_{1}+i c_{2}\right), \quad a_{2}=\frac{1}{\sqrt{2}}\left(c_{2}+i c_{1}\right) .
$$

So that $c_{i}=\partial / \partial c_{i}^{*}$ and $\left[c_{i}, c_{j}^{*}\right]=\delta_{i j}$. In terms of these we have

$$
\begin{aligned}
& L_{12}=\frac{i}{2}\left(c_{1}^{*} c_{2}-c_{2}^{*} c_{1}\right) \\
& L_{13}=\frac{\varepsilon}{2}\left(c_{1}^{*} c_{2}+c_{2}^{*} c_{1}\right) \\
& L_{23}=\frac{\varepsilon}{2}\left(c_{1}^{*} c_{1}-c_{2}^{*} c_{2}\right) .
\end{aligned}
$$

Thus we have an automorphism of the Lie algebra

$$
\begin{aligned}
& L_{12} \rightarrow \frac{1}{\varepsilon} L_{23} \\
& L_{13} \rightarrow L_{13} \\
& L_{23} \rightarrow-\varepsilon L_{12} .
\end{aligned}
$$

Thus, for compact case, $\varepsilon=1, L_{23}$ and $L_{12}$ have obviously the same spectrum, but for the non-compact case, the representation with $L_{23}$ having real continuous spectrum is the same as the representation with $L_{12}$ diagonal and having a purely imaginary spectrum.

In the representation (3.3), $L_{23}$ is diagonal, and we use the same method as in Section II now to the states

The states $|\lambda\rangle$ are given by

$$
L_{23}|\lambda\rangle=\lambda|\lambda\rangle
$$

$$
|\lambda\rangle=A_{\lambda} c_{1}^{* \Phi+\varepsilon \lambda} c_{2}^{* \Phi-\varepsilon \lambda}
$$

or by

$$
|\lambda\rangle=A_{\lambda}\left(\frac{a_{1}^{*}+i a_{2}^{*}}{\sqrt{2}}\right)^{\Phi+\varepsilon \lambda}\left(\frac{a_{2}^{*}+i a_{1}^{*}}{\sqrt{2}}\right)^{\Phi-\varepsilon \lambda}
$$

Indeed, under $U$ [Eq. (2.8)] applied to $\left(\begin{array}{c}a_{3}^{*} \\ a_{2}^{*}\end{array}\right)$ we find

i.e.

$$
e^{i \xi L_{2 \mathrm{~s}}}|\lambda\rangle=e^{-i \varepsilon \frac{\xi}{2}(\Phi+\varepsilon \lambda)} e^{i \varepsilon \frac{\xi}{2}(\Phi-8 \lambda)}|\lambda\rangle=e^{-i\left(-g_{\mathrm{s}}\right) \xi \lambda}|\lambda\rangle
$$

$$
L_{23}|\lambda\rangle=-\left(-g_{33}\right) \lambda|\lambda\rangle
$$

or, under $L_{12}$-rotation applied on $\left(\begin{array}{l}c_{1}^{*} \\ c_{2}^{*}\end{array}\right)$

i.e.

$$
e^{i \mu L_{12}}|\lambda\rangle=e^{-i \frac{\mu}{2}(\Phi+\varepsilon \lambda)} e^{i \frac{\mu}{2}(\Phi-\varepsilon \lambda)}|\lambda\rangle
$$

$$
L_{12}|\lambda\rangle=-\varepsilon \lambda|\lambda\rangle
$$




\section{(1) Transformation between Discrete and Continuous Basis}

We first expand the $L_{23}$-states in terms of the $L_{12}$-states. The latter are

$$
|\Phi, m\rangle=A_{m} a_{1}^{* \Phi_{+m}} a_{2}^{* \Phi-m} \text {. }
$$

From (3.7) we obtain by expansion

$$
|\lambda\rangle=\frac{A_{\lambda}}{2^{\Phi}} \sum_{N, N^{\prime}}\left(\begin{array}{c}
\Phi+\varepsilon \lambda \\
N
\end{array}\right)\left(\begin{array}{c}
\Phi-\varepsilon \lambda \\
N^{\prime}
\end{array}\right)\left(a_{1}^{*}\right)^{N+N^{\prime}}\left(a_{2}^{*}\right)^{2 \Phi-N-N^{\prime}}(i)^{\Phi+\varepsilon \lambda-N+N^{\prime}}
$$

let $N+N^{\prime}=\Phi+m$,

$$
\begin{aligned}
|\lambda\rangle & =\frac{A_{\lambda}}{2 \Phi} \sum\left(\begin{array}{c}
\Phi+\varepsilon \lambda \\
\Phi-N^{\prime}+m
\end{array}\right)\left(\begin{array}{c}
\Phi-\varepsilon \lambda \\
N^{\prime}
\end{array}\right) i^{\varepsilon \lambda-m+2 N^{\prime}} a_{1}^{* \Phi+m} a_{2}^{* \Phi-m} \\
& =\sum_{m} \frac{A_{\lambda}}{2 \Phi A_{m}} i^{\varepsilon \lambda-m}|m\rangle \sum_{N^{\prime}}\left(\begin{array}{c}
\Phi+\varepsilon \lambda \\
\Phi-N^{\prime}+m
\end{array}\right)\left(\begin{array}{c}
\Phi-\varepsilon \lambda \\
N^{\prime}
\end{array}\right)(-1)^{N^{\prime}} .
\end{aligned}
$$

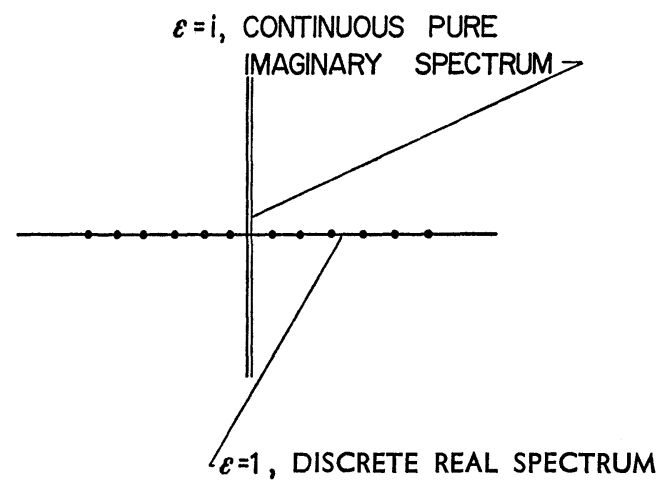

Fig. 1. Spectrum of $L_{23}$ in the compact and non-compact cases

If we introduce the hypergeometric function, we get

$$
\begin{aligned}
\langle m \mid \lambda\rangle= & 2^{-\Phi} \frac{A_{\lambda}}{A_{m}} i^{(\varepsilon \lambda-m)} \frac{\Gamma(\Phi+\varepsilon \lambda+1)}{\Gamma(\varepsilon \lambda-m+1) \Gamma(\Phi+m+1)} \\
& \cdot F(-\Phi-m,-\Phi+\varepsilon \lambda, \varepsilon \lambda-m+1 ;-1) .
\end{aligned}
$$

In particular, in the compact case, $\varepsilon=1, \lambda=l=$ integer,

$$
\begin{aligned}
\langle m \mid l\rangle= & 2^{-\Phi} \frac{A_{l}}{A_{m}} i^{l-m} \frac{\Gamma(\Phi+l+1)}{\Gamma(\Phi+m+1) \Gamma(l-m+1)} \\
& \cdot F(-\Phi-m,-\Phi+l, l-m+1 ;-1)
\end{aligned}
$$

which must be equal to the matrix element of a rotation by $\pi / 2$ :

$$
\alpha=\beta=\frac{1}{\sqrt{2}},-\beta^{2} / \alpha^{2}=-1 .
$$

Indeed we find from (2.11) that (3.11) reduces to

$$
\langle m \mid l\rangle=D\left(e^{i \frac{\pi}{2} L_{13}}\right)_{m l} \text {. }
$$

In the $\lambda$-plane the discrete and the continuous spectra are shown in Fig. 1. 
(2) Matrix Elements of Finite Transformation in the Continuous Basis

We consider again the $L_{\mathbf{2}}$-basis (3.5). It is sufficient to determine the matrix elements of an $L_{12}$-rotation, $e^{i \mu\left(L_{12}\right)}$. In the fundamental representation, from (2.5)

Thus from (3.7)

$$
\begin{aligned}
W & =e^{i \theta L_{12}}=\left(\begin{array}{cc}
e^{i \theta / 2} & \\
e^{-i \theta / 2}
\end{array}\right)=\left(\begin{array}{c}
\alpha \\
\bar{\alpha}
\end{array}\right) \\
W^{-1} & =\left(\begin{array}{c}
\bar{\alpha} \\
\alpha
\end{array}\right),|\alpha|=1 .
\end{aligned}
$$

where we have put

$$
\begin{aligned}
D(W)|\lambda\rangle & =\frac{A_{\lambda}}{2^{\Phi}}\left(\bar{\alpha} a_{1}^{*}+i \alpha a_{2}^{*}\right)^{\Phi+\varepsilon \lambda}\left(\alpha a_{2}^{*}+i \bar{\alpha} a_{1}^{*}\right)^{\Phi-\varepsilon \lambda} \\
& =A_{\lambda}\left(a c_{1}^{*}-b c_{2}^{*}\right)^{\Phi+\varepsilon \lambda}\left(b c_{1}^{*}+a c_{2}^{*}\right)^{\Phi-\varepsilon \lambda}
\end{aligned}
$$

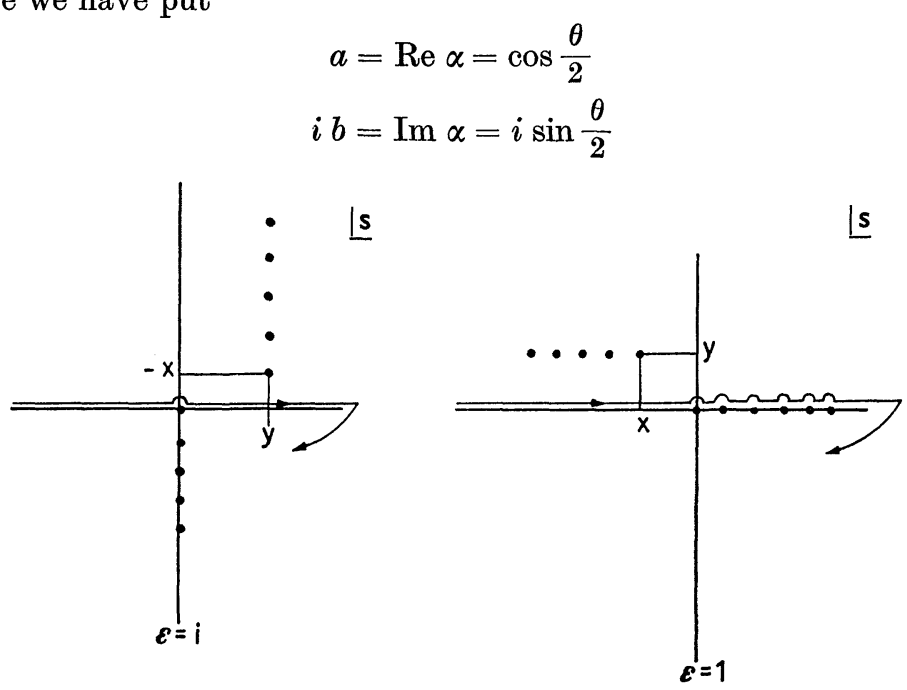

Fig. 2. Contours of integrations and poles of the integrand for the general binomial expansion (3.15), for $x<0, y>0$

We must expand now (3.14) in terms of the continuous basis states $|\mu\rangle$. In the case of the continuous basis we use the integral representation of the binomial expansion [11]

$(A+B)^{z}=[2 \pi i \Gamma(-z)]^{-1} \int_{-\frac{i}{8} \infty}^{\frac{i}{8} \infty} \Gamma(-z+\varepsilon s) \Gamma(-\varepsilon s)(B)^{\varepsilon s} A^{z-\varepsilon s} d(\varepsilon s)$

valid for

$$
|B|<|A|,|\arg (-B \mid A)|<\pi .
$$

Here $\Gamma(-z+\varepsilon s)(z=x+i y)$ has poles at: $s=y+i(n-x), n=0,1,2, \ldots$, i.e. in the upper half plane, for $x<0, y>0$ for non compact case and at $s=-n+x+i y, n=0,1,2, \ldots$, along the real axis for compact case. $\Gamma(-\varepsilon s)$ has poles also at $s=-i n$ and $s=n$, respectively (Fig. 2). 
As sum of residues, equation (3.15) reduces to

$$
(A+B)^{z}=\frac{1}{\Gamma(-z)} \sum_{N}(\Gamma(N-z) / \Gamma(N+1)) B^{N} A^{z-N},
$$

i.e. the usual binomial expansion. We now apply (3.15) to (3.14):

$$
\begin{aligned}
D(W)|\lambda\rangle= & A_{\lambda} \frac{1}{2 \pi \Gamma(-\Phi-\varepsilon \lambda)} \frac{1}{2 \pi \Gamma(-\Phi+\varepsilon \lambda)} \\
& \cdot i^{-2} \int_{-\frac{i}{\varepsilon} \infty}^{\frac{i}{\varepsilon} \infty} d(\varepsilon s) d\left(\varepsilon s^{\prime}\right) \Gamma(-\Phi-\varepsilon \lambda+\varepsilon s) \Gamma(-\varepsilon s) \\
& \cdot \Gamma\left(-\Phi+\varepsilon \lambda+\varepsilon s^{\prime}\right) \Gamma\left(-\varepsilon s^{\prime}\right)(-b)^{\varepsilon s} \\
& \cdot c_{2}^{* \varepsilon s} a^{\Phi+\varepsilon \lambda-\varepsilon s} c_{1}^{* \Phi+\varepsilon \lambda-8 s} b^{\varepsilon s^{\prime}} c_{1}^{* \varepsilon s^{\prime}} a^{\Phi-\varepsilon \lambda-\varepsilon s^{\prime}} c_{2}^{* \Phi-\varepsilon \lambda-8 s^{\prime}} .
\end{aligned}
$$

The last line can be rewritten to give

Thus, letting

$$
(-b)^{\varepsilon s} b^{\varepsilon s^{\prime}} a^{2 \Phi-\varepsilon s-\varepsilon s^{\prime}} c_{1}^{* \Phi+\varepsilon \lambda-\varepsilon s+\varepsilon s^{\prime}} c_{2}^{* \Phi-\varepsilon \lambda+\varepsilon \varepsilon-\varepsilon s^{\prime}} .
$$

or

$$
\varepsilon \mu=\varepsilon \lambda-\varepsilon s+\varepsilon s^{\prime}
$$

$$
s^{\prime}=\mu-\lambda+s
$$

so that $d s^{\prime}=d \mu$, and integrating over $d s d \mu$ instead of $d s d s^{\prime}$, we obtain:

$$
\begin{aligned}
D(W)|\lambda\rangle= & \int_{-\frac{i}{\varepsilon} \infty}^{\frac{i}{\varepsilon} \infty} \varepsilon d \mu \frac{A_{\lambda}}{A_{\mu}} \frac{|\mu\rangle}{2 \pi i \Gamma(-\Phi-\varepsilon \lambda) \Gamma(-\Phi+\varepsilon \lambda)}(b)^{\varepsilon(\mu-\lambda) a^{2 \Phi-\varepsilon(\mu-\lambda)}} \\
& \cdot \frac{1}{2 \pi i} \int_{-\frac{i}{8} \infty}^{+\frac{i}{8} \infty} d(\varepsilon s) \Gamma(-\Phi-\varepsilon \lambda+\varepsilon s) \Gamma(-\varepsilon s) \\
& \cdot \Gamma(-\Phi+\varepsilon \mu+\varepsilon s) \Gamma(\varepsilon \lambda-\varepsilon \mu-\varepsilon s)\left(\frac{-b^{2}}{a^{2}}\right)^{\varepsilon s}
\end{aligned}
$$

The value of the second integral is $-2 \pi i \Sigma$ (Residues) of poles (in the lower half plane, non-compact case) at (Fig. 3).

$$
\varepsilon s=N, \quad \text { and } \quad \varepsilon s=N+\varepsilon \lambda-\varepsilon \mu, N=0,1,2,3, \ldots
$$

For the first set of poles we get for the second integral

$$
\begin{aligned}
\sum_{N=}^{\infty} & \frac{\Gamma(-\Phi-\varepsilon \lambda+N) \Gamma(-\Phi+\varepsilon \mu+N) \Gamma(\varepsilon \lambda-\varepsilon \mu-N)}{\Gamma(N+1)}\left(\frac{-b^{2}}{a^{2}}\right)^{N}(-1)^{N} \\
= & \frac{\pi}{\sin \pi(\varepsilon \lambda-\varepsilon \mu)} \frac{\Gamma(-\Phi-\varepsilon \lambda) \Gamma(\Phi+\varepsilon \mu)}{\Gamma(1-\varepsilon \lambda+\varepsilon \mu)} \\
& \cdot F\left(-\Phi-\varepsilon \lambda,-\Phi+\varepsilon \lambda, 1-\varepsilon \lambda+\varepsilon \mu, \frac{-b^{2}}{a^{2}}\right) .
\end{aligned}
$$


Similarly, for the second set of poles we obtain

$$
\begin{aligned}
& \sum_{N=0}^{\infty} \frac{\Gamma(-\Phi-\varepsilon \mu+N) \Gamma(\varepsilon \mu-\varepsilon \lambda-N) \Gamma(-\Phi+\varepsilon \lambda+N)}{\Gamma(N+1)} \\
& \cdot(-1)^{N}\left(-b^{2} / a^{2}\right)^{N+\varepsilon \lambda-\varepsilon \mu}=\frac{\pi\left(-b^{2} / a^{2}\right)^{\varepsilon \lambda-\varepsilon \mu}}{\sin \pi(\varepsilon \mu-\varepsilon \lambda)} \frac{\Gamma(-\Phi-\varepsilon \mu) \Gamma(-\Phi+\varepsilon \lambda)}{\Gamma(1-\varepsilon \mu+\varepsilon \lambda)} \\
& \cdot F\left(-\Phi-\varepsilon \mu,-\Phi+\varepsilon \lambda, 1-\varepsilon \mu+\varepsilon \lambda ; \frac{-b^{2}}{a^{2}}\right) \text {. }
\end{aligned}
$$

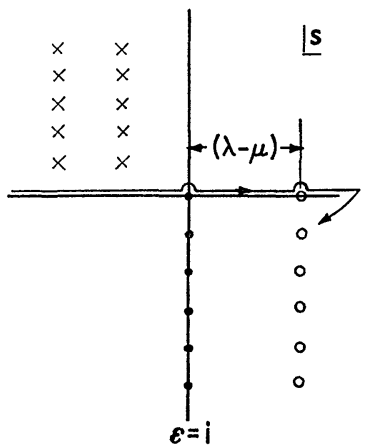

Fig. 3. Contours of integrations for Eq. (3.17). poles of $\Gamma(-\varepsilon s)$; O poles of $\Gamma(\varepsilon \lambda-\varepsilon \mu-\varepsilon s) ; \times$ poles of $\Gamma(-\Phi-\varepsilon \lambda+\varepsilon s)$ and $\Gamma(-\Phi+\varepsilon \mu+\varepsilon s)$. For $\varepsilon=1$, the contour has to avoid the poles $\times$, and the contributions of the poles of type $\bigcirc$ and $O$, that coincide, exactly cancel

In the compact case, the poles lie on the real axis and the contribution of the poles coinciding (see Fig. 2) exactly cancel so that we obtain only a finite sum as it should be. Thus inserting these into (3.17) we get

$$
\begin{aligned}
& D(W)|\lambda\rangle=\int_{-\frac{i}{8} \infty}^{+\frac{i}{8} \infty} \frac{A_{\lambda}}{A_{\mu}}|\mu\rangle d \mu \frac{\varepsilon}{2 \pi i} b^{\varepsilon(\mu-\lambda)} a^{2 \Phi-\varepsilon(\mu-\lambda)} \\
& \quad \cdot\left\{\frac{\Gamma(-\Phi+\varepsilon \mu) \Gamma(\varepsilon \lambda-\varepsilon \mu)}{\Gamma(-\Phi+\varepsilon \lambda)} F\left(-\Phi-\varepsilon \lambda,-\Phi+\varepsilon \mu, 1-\varepsilon \lambda+\varepsilon \mu ; \frac{-b^{2}}{a^{2}}\right)\right. \\
& +\left(\frac{-b^{2}}{a^{2}}\right)^{\varepsilon \lambda-\varepsilon \mu} \frac{\Gamma(-\Phi-\varepsilon \mu) \Gamma(\varepsilon \mu-\varepsilon \lambda)}{\Gamma(-\Phi-\varepsilon \lambda)} \\
& \left.\quad \cdot F\left(-\Phi-\varepsilon \mu,-\Phi+\varepsilon \lambda, 1-\varepsilon \mu+\varepsilon \lambda ; \frac{-b^{2}}{a^{2}}\right)\right\} .
\end{aligned}
$$

Note: The phase of $(-b)$ is chosen to be $e^{i \pi}$ thoughout.

In the non-compact case we can then write the matrix elements in the 
symmetric form

$$
\begin{aligned}
D(W)_{\mu \lambda} & =\frac{A_{\lambda}}{A_{\mu}} \frac{1}{2 \pi} e^{-i \pi \frac{\varepsilon \mu-\varepsilon \lambda}{2}} a^{2 \Phi} \\
& \cdot\left\{\left(\frac{-b^{2}}{a^{2}}\right)^{\frac{\varepsilon \mu-\varepsilon \lambda}{2}} \frac{\Gamma(-\Phi+\varepsilon \mu) \Gamma(\varepsilon \lambda-\varepsilon \mu)}{\Gamma(-\Phi+\varepsilon \mu)}\right. \\
& \cdot F\left(-\Phi-\varepsilon \lambda,-\Phi+\varepsilon \mu, 1-\varepsilon \lambda+\varepsilon \mu ; \frac{-b^{2}}{a^{2}}\right) \\
& +\left(\frac{-b^{2}}{a^{2}}\right)^{\frac{\varepsilon \lambda-\varepsilon \mu}{2}} \frac{\Gamma(-\Phi-\varepsilon \mu) \Gamma(\varepsilon \mu-\varepsilon \lambda)}{\Gamma(-\Phi-\varepsilon \lambda)} \\
& \left.\cdot F\left(-\Phi+\varepsilon \lambda,-\Phi-\varepsilon \mu, 1-\varepsilon \mu+\varepsilon \lambda ; \frac{-b^{2}}{a^{2}}\right)\right\} .
\end{aligned}
$$

In the compact case, the integral in (3.18) has to reduce to a finite sum. This indeed is the case: We get contributions from the poles of

$$
\Gamma(-\Phi+\mu) \text { and } \Gamma(-\Phi-\mu) \text {. }
$$

For $-\Phi+\mu=$ negative integer, for example, we have from (3.18) (writing $\lambda=m, \mu=m^{\prime}$ ), for $m<m^{\prime}$

$$
\begin{aligned}
D(W)|m\rangle= & \frac{A_{m}}{A_{m^{\prime}}} b^{m^{\prime}-m} a^{2 \Phi-\left(m^{\prime}-m\right)}\left|m^{\prime}\right\rangle \\
& \cdot \frac{(-1)^{\Phi-m^{\prime}}}{\Gamma\left(\Phi-m^{\prime}+1\right)} \frac{\Gamma\left(m-m^{\prime}\right)}{\Gamma(-\Phi+m)} \\
& \cdot F\left(-\Phi-m,-\Phi+m^{\prime}, 1-m+m^{\prime} ; \frac{-b^{2}}{a^{2}}\right)
\end{aligned}
$$

or,

$$
\begin{aligned}
\left\langle m^{\prime}|D(W)| m\right\rangle & =\frac{A_{m}}{A_{m^{\prime}}} b^{m^{\prime}-m} a^{2 \Phi-m^{\prime}+m} \frac{\Gamma(1+\Phi-m)}{\Gamma\left(1+\Phi-m^{\prime}\right)} \frac{(-1)^{\Phi-m^{\prime}}}{\Gamma\left(1+m^{\prime}-m\right)} \\
& \cdot \frac{\sin \pi(\Phi-m)}{\sin \pi\left(m^{\prime}-m\right)} F\left(-\Phi+m^{\prime},-\Phi-m, 1+m^{\prime}-m ; \frac{-b^{2}}{a^{2}}\right)
\end{aligned}
$$

which agrees with (2.12).

The second term in the parenthesis is obtained from the first by $\mu \rightarrow-\mu, \lambda \rightarrow-\lambda$. Thus we have the relation

$$
D_{-\mu,-\gamma}=-\frac{A_{-\lambda}}{A_{\lambda}} \frac{A_{\mu}}{A_{-\mu}} D_{\mu, \lambda} \text {. }
$$

Normalization of States. The states $|\lambda\rangle$ must be correctly normalized to

This means

$$
\langle\mu \mid \lambda\rangle=\delta(\mu-\lambda), \quad \text { or } \delta_{\mu \lambda} \text {. }
$$

$$
D_{\mu \lambda}(0)=\delta(\mu-\lambda), \text { or } \delta_{\mu \lambda}
$$

as $b \rightarrow 0, a \rightarrow 1$. The discrete case is easy to see.

Letting $-b / a=\eta, \mu-\lambda=x$, we have for $\mu \rightarrow \lambda$

$$
\lim _{\mu \rightarrow \lambda} D_{\mu \lambda}(b \rightarrow 0)=\lim _{\eta \rightarrow 0} \frac{1}{2 \pi} \eta^{\varepsilon \varkappa}\left[\Gamma(-\varepsilon x)+\eta^{-2 \varepsilon x} \Gamma(\varepsilon x)\right] .
$$


The limit gives the desired result because of the identity

$$
\lim _{\eta \rightarrow 0}\left[\eta^{i x} \Gamma(i x)-\eta^{-i x} \Gamma(-i x)\right]=2 \pi \delta(x) .
$$

(3) Calculation of the Normalization Constants

We have the requirement of unitarity so that

$$
\int f_{\lambda}^{*} f_{\lambda} d \lambda=\int\left(D(W) f_{\lambda}\right) *\left(D(W) f_{\lambda}\right) d \lambda .
$$

In the case of $\langle m \mid \lambda\rangle$ we use

$$
\sum_{m} f_{m}^{*} f_{m}=\sum_{m}\left(D f_{m}\right)^{*}\left(D f_{m}\right)=\int \sum_{m}\left\langle m\left|D^{+}\right| \lambda\right\rangle\langle\lambda|D| m\rangle d \lambda .
$$

Consider the expression

$$
\left\{\left|a_{1}^{*}+i a_{2}^{*}\right|^{2}+\left|a_{2}^{*}+i a_{1}^{*}\right|^{2}\right\}^{2 \Phi}=\left\{\left|a_{1}^{*}\right|^{2}+\left|a_{2}^{*}\right|^{2}\right\}^{2 \Phi} .
$$

The right hand side gives

$$
\begin{aligned}
& \sum_{m=0}^{\infty} \frac{\Gamma(2 \Phi+1)}{\Gamma(\Phi-m+1) \Gamma(\Phi+m+1)}\left|a_{1}^{*}\right|^{2(\Phi+m)}\left|a_{2}^{*}\right|^{2(\Phi-m)} \\
& \quad=\sum_{m}\left|A_{m}\right|^{2}\left|a_{1}^{*}\right|^{2(\Phi+m)}\left|a_{2}^{*}\right|^{2(\Phi-m)}=\sum_{m} f_{m}^{*} f_{m} .
\end{aligned}
$$

Hence $\left|A_{m}\right|^{2}$ can be chosen to be

$$
\left|A_{m}\right|^{2}=\frac{\Gamma(2 \Phi+1)}{\Gamma(\Phi-m+1) \Gamma(\Phi+m+1)} .
$$

Both $\Gamma(2 \Phi+1), \Gamma(-m+\Phi+1)$ have poles, these cancel to give

$$
\left|A_{m}\right|^{2}=\frac{(-1)^{\Phi+m} \Gamma(m-\Phi)}{\Gamma(\Phi+m+1) \Gamma(-2 \Phi)} .
$$

The left hand side of (3.23) gives

$$
\begin{gathered}
\frac{1}{2 \pi} \int_{-\infty}^{+\infty} \frac{\Gamma(-\Phi+\varepsilon \lambda) \Gamma(-\Phi-\varepsilon \lambda)}{\Gamma(-2 \Phi)} 2^{\Phi}\left|c_{1}^{*}\right|^{2(\Phi+\varepsilon \lambda)}\left|c_{2}^{*}\right|^{2(\Phi-\varepsilon \lambda)} d \lambda \\
=\int\left|A_{\lambda}\right|^{2}\left|c_{1}^{*}\right|^{2(\Phi+\varepsilon \lambda)}\left|c_{2}^{*}\right|^{2(\Phi-\varepsilon \lambda)} d \lambda \\
\text { and }\left|A_{\lambda}\right|^{2}=\frac{2^{\Phi}}{2 \pi} \frac{\Gamma(-\Phi+\varepsilon \lambda) \Gamma(-\Phi-\varepsilon \lambda)}{\Gamma(-2 \Phi)}
\end{gathered}
$$

We see that $\frac{\left|A_{\lambda}\right|^{2}}{\left|A_{\mu}\right|^{2}}$ correctly reduces to $\frac{\left|A_{m}\right|^{2}}{\left|A_{m^{\prime}}\right|^{2}}$ for the compact case $\varepsilon=+1$. Note that only this ratio is uniquely determined.

From (3.19) and (3.25) we have finally

$$
D_{-\mu,-\lambda}=-D_{\mu, \lambda} \text {. }
$$

\section{Conclusions}

It is well-known that in the case of the continuous basis (the set of eigenvectors of a non-compact generator), the Lie algebra operators $L^{ \pm}$ transform the state $|\lambda\rangle$ into states $|\lambda \pm i\rangle$ that are not among the basis 
states and must be expanded in terms of the basis states. We have used the algebraic forms of these states in terms of creation and annihilation operators with complex exponents to make these required expansions from the beginning. We have arrived thus at general formulas, Eq. (3.10) and Eq. (3.18), valid for both compact and non-compact cases, which reduce correctly to finite sums in the compact case.

\section{References}

1. KuRIYAN, J. G., N. MuKunda, and E. C. G. Sudarshan: Institute for Advanced Study preprint 1967.

2. MuKunda, N.: Syracuse University preprint 1967.

3. BARUT, A. O.: Unified algebraic construction of representations of compact and non-compact Lie Algebras and Lie groups. In: Lectures in theoretical physics, Vol. IXA, p. 125-171. New York: Gordon \& Breach 1967.

4. Sciarrino, A., and M. Toller: University of Rome preprint, No. 108, 1966.

5. Mukunda, N.: Syracuse University preprints, 1967.

6. RÜHL, W.: Commun. Math. Phys. 6, 312 (1967)

7. Akyeampong, D. A., J. F. Boyce, and M. A. Rashid: Nuovo Cimento (in press).

8. Barut, A. O., and C. Fronsdal: Proc. Roy. Soc. (London) Ser. A 287, 532 (1965).

9. Holman, W. J., and L. C. Biedenharn: Ann. Phys. 39, 1 (1966).

10. BargmanN, V.: Ann. Math. 48, 568 (1947).

11. WhitTaker, E. T., and G. N. Watson: A course of modern analysis, Fourth Edition, p. 289. London: Cambridge University Press, 1965.

Prof. A. O. Barut

Department of Physics

University of Colorado

Boulder, Colorado 80302

USA
Mr. E. C. PHسLLIPS

Department of Mathematics

University of Colorado

Boulder, Colorado 80302

USA 